\title{
Nutritional status of parasitised individuals with Toxocara canis in the State of Mexico
}

\author{
N. Ramírez Durán ${ }^{1}$, R. Valdés Ramos ${ }^{1}$, C. Romero Nuñez ${ }^{1}$, G. Vázquez García ${ }^{2}$, \\ S. Y. Yañez Arteaga ${ }^{2}$, G. D. Mendoza Martínez ${ }^{2}$ \\ ${ }^{1}$ Center for Research and Graduate Studies in Health Sciences, Faculty of Medicine, Universidad Autónoma del Estado \\ de México and ${ }^{2}$ Universidad Autónoma Metropolitana, Unidad Xochimilco, Medicina Veterinaria y Zootecnia, DF México
}

Toxocarosis is entheroparasitosis frequently present in dogs and cats which is maintained in the environment by the infestation and reinfestation of the hosts, by the ingestion of food and soil contaminated with larvated eggs, ingestion of larvae in tissue of paratenic larvae (rats and birds), by transplacentary migration of a female dog to their fetus, transmammary passage in milk, or ingestion of larvae or faeces from puppets infested ${ }^{(1)}$. Human subjects, mainly children, are infested by the accidental ingestion of embryonated eggs from the soil contaminated by the dogs, and even when most of the seropositive patients may be asymptomatic, the parasite may cause fever, hepatomegaly, esplenomegaly, hypergammaglobulinaemia, eosinophilia, adenopathies and disorders of central nervous system, myocardium, eyes, skin, respiratory symptoms and even as a fatal disease ${ }^{(2,3)}$.

The aim of the present study was to analyse the relationship of nutritional status in Toxocara canis infested children and adolescents in the State of Mexico. A study comprising 108 patients between 2 and 16 years of age, both male and female (56 men and 52 women) from the State of Mexico was carried out. Their T. canis antibodies' levels were measured by means of an ELISA test. The BMI was evaluated in order to assess nutritional status. We used a chi-square and risk test (OR) in order to compare the difference between groups and the relationship between nutritional status and parasitosis. A regression analysis was also conducted between antibody presence and BMI.

Nutritional status of patients and its relationship with $T$. canis serology

\begin{tabular}{lcccrr}
\hline Nutritional status & $\begin{array}{c}\text { Positives } \\
(n \text { 25 })\end{array}$ & $\begin{array}{c}\text { Negatives } \\
(n \text { 83) }\end{array}$ & Chi-square & OR & $P$ \\
\hline Normal & 12 & 41 & 0.01 & 0.94 & 0.90 \\
Low weight/malnutrition & 9 & 23 & 0.63 & 1.43 & 0.42 \\
Overweight/obesity & 4 & 19 & 0.54 & 0.64 & 0.46 \\
\hline
\end{tabular}

According to standards established by the World Health Organization (2004), 49\% of the participants had a normal BMI; $30 \%$ showed low weight, first- and second-degree malnutrition; and the remaining $21 \%$ showed overweight and first-degree obesity. Twenty-five individuals (fourteen males and eleven females) between 2 and 14 years of age presented antibodies for T. canis larva migrans. From these parasitised children and teenagers $48 \%$ showed a normal BMI; $36 \%$ showed underweight and first- and second-degree malnutrition; and $16 \%$ showed overweight and first-degree obesity.

Nutritional status of patients and nutritional status of T. canis positives

\begin{tabular}{lcccccc}
\hline & & & & \multicolumn{2}{c}{ Toxocara canis positives } \\
\cline { 5 - 7 } Nutritional status & $\%$ & BMI (average) & Men & Women & Men & Women \\
\hline Low weight/malnutrition & 30 & 17.7823 & 16 & 16 & 4 & 5 \\
Normal & 49 & 22.24 & 27 & 26 & 8 & 4 \\
Overweight/obesity & 21 & 27.8 & 13 & 10 & 2 & 2 \\
\hline
\end{tabular}

Nutritional status was not associated with toxocariasis serology; risk factors (RF) are low, indicating that other factors may be more important in the presence of the disease. The lack of a relation between BMI and parasitosis was confirmed by the null correlation between seropositivity and BMI $(r=0.08)$. It can be concluded that nutritional status is not associated with seropositivity for Toxocara. Parasitic infections are highly prevalent in populations of developing countries, affecting mostly those groups with higher nutritional deficit; therefore it is intended to extend the current study with the purpose of relating toxocariasis pathophysiology with the nutritional status of patients.

1. Archelli S \& Kozubsky L (2008) Acta Bioquím Clín Latinoam. 42, 379-384.

2. Camparoto M, Fulan B, Colli M et al. (2008) Gen Mol Res 7, 444-450.

3. Rubinsky-Elefant G, Hirata CE, Yamamoto JH et al. (2010) Ann Trop Med Parasitol 104, 3-23. 